\title{
Combined Treatment with 1,550-nm Fractional Erbium:Glass Fiber and 2,940-nm Fractional Erbium:YAG Lasers for Atrophic Scars
}

\author{
Jiehoon $\mathrm{Kim}^{1}$ \\ Hwa Jung Ryu ${ }^{2}$ \\ Sung Bin $\mathrm{Cho}^{3,4}$
}

${ }^{1}$ Dr. Kim's Skin \& Laser Clinic, Dermatology,
Suwon, Korea
2Department of Dermatology, College of
Medicine, Korea University Ansan Hospital,
Ansan, Korea
${ }^{3}$ Department of Dermatology, International St.
Mary's Hospital, Catholic Kwandong University
College of Medicine, Incheon, Korea
${ }^{4}$ Kangskin Sillim Dermatology Clinic, Seoul,
Korea

Received October 23, 2016

Accepted November 2, 2016

Correspondence
Sung Bin Cho
Department of Dermatology, International St.
Mary's Hospital, Catholic Kwandong University
College of Medicine, 25 Simgok-ro, 100beon-gil,
Seo-gu, Incheon 22711, Korea
Tel.: +82-32-290-3141
Fax: +82-32-290-3142
E-maill: drsbcholagmail.com
(C) Korean Society for Laser Medicine and Surgery
@c This is an open access article distributed under the
terms of the Creative Commons Attribution Non-
Commercial License (http://creativecommons.org/
licenses/by-nc/4.0) which permits unrestricted non-
commercial use, distribution, and reproduction in any
medium, provided the original work is properly cited.

\begin{abstract}
Atrophic scars may arise from various causes, including herpes simplex virus (HSV) infection and burns. In treating atrophic scars, the application of individual treatments alone has proven to be ineffective. Meanwhile, however, combined treatment methods have been found to offer synergistic effects for better treatment results with minimal adverse reactions. In this report, we describe our experiences in treating two patients with atrophic scars: One with HSV-induced atrophic scars on the cheek, and the other with burn-induced atrophic and dyspigmented scars along the lower leg. Patients were effectively treated with combined treatment using a non-ablative 1,550-nm fractional erbium (Er):glass fiber laser and an ablative 2,940-nm fractional Er:yttrium aluminum garnet laser. We suggest that combined therapy with different kinds of fractional lasers may provide synergistic therapeutic effects in treating Asian patients with atrophic scars.
\end{abstract}

\section{Key words}

Atrophic scar; Herpes simplex virus; Burn; 1,550-nm fractional erbium:glass fiber laser; 2,940-nm fractional erbium:YAG laser 


\section{INTRODUCTION}

Atrophic scars originate from inflammatory acne lesions; furuncles and carbuncles; small pox, chicken pox, herpes simplex virus (HSV), and herpes zoster virus infections; and physical trauma or burns. These scars are indicated for treatment with topical agents, needling therapy, subcision (subcuticular incision), mechanical- or laserassisted skin resurfacing procedures, chemical peeling, chemical reconstruction of skin scars, noninvasive or invasive radiofrequency treatment, and non-ablative or ablative fractional laser treatment. ${ }^{1-3}$ None of these, however, have been found to be remarkably effective in treating atrophic scars on their own.

Laser-assisted skin resurfacing was developed as a means to safely ablate the surface of the skin for effective removal of targeted lesions and regeneration of skin structures. ${ }^{4}$ However, prolonged wound healing thereafter and high risks of developing post-inflammatory hyperpigmentation and erythema have limited the use of ablative lasers in Asian patients. ${ }^{4}$ For these reasons, fractional laser systems have been more widely used to treat various dermatologic disorders in Asian patients, although several sessions of laser treatments are usually needed for satisfactory clinical outcomes, compared to conventional resurfacing lasers. Fractional laser systems generate microscopic zones of photothermal reactions, wherein the surrounding skin is left intact, allowing for a shorter duration of post-laser wound healing and a reduced risk of adverse events. ${ }^{4}$ Accordingly, fractional lasers can be more effectively and safely combined with different treatment modalities or other fractional energy delivery systems for treating lesions of the skin.

In this report, we describe our experiences with using a non-ablative 1,550-nm fractional erbium (Er):glass fiber laser and an ablative 2,940-nm fractional Er:yttrium aluminum garnet (YAG) laser to treat atrophic scars.

\section{CASE REPORTS}

\section{Case 1}

A 24-year-old male patient visited our clinic presenting with atrophic scars on his left cheek. The lesions had developed after HSV infection approximately 15 years ago. On his first visitation, round to ovoid atrophic scars with brownish pigmentation in a curved to linear arrangement were documented along the cheek (Fig. 1A). After obtaining written informed consent, two sessions of combined treatment using a 1,550-nm fractional Er:glass fiber laser (MOSAIC HP; Lutronic Corp., Goyang, Koreal and a 2,940nm fractional Er:YAG laser (ACTION II; Lutronic Corp.) were performed at 4-week intervals. To begin, topical EMLA cream leutectic mixture of $2.5 \%$ lidocaine $\mathrm{HCl}$ and 2.5\% prilocaine; AstraZeneca AB, Södertälje, Sweden) for local anesthesia was applied on the lesions for 1 hour and cleansed with 70\% ethanol. Then, treatment with the nonablative 1,550-nm fractional erbium Er:glass fiber laser was performed first with the settings of $20 \mathrm{~mJ}$ on the entire face and 33-35 $\mathrm{mJ}$ on the atrophic lesions using a dynamic operating mode and a $4 \mathrm{~cm} \times 4 \mathrm{~cm}$ tip. Immediately after the non-ablative treatment, ablative 2,940-nm fractional Er:YAG laser treatment was delivered to the lesions

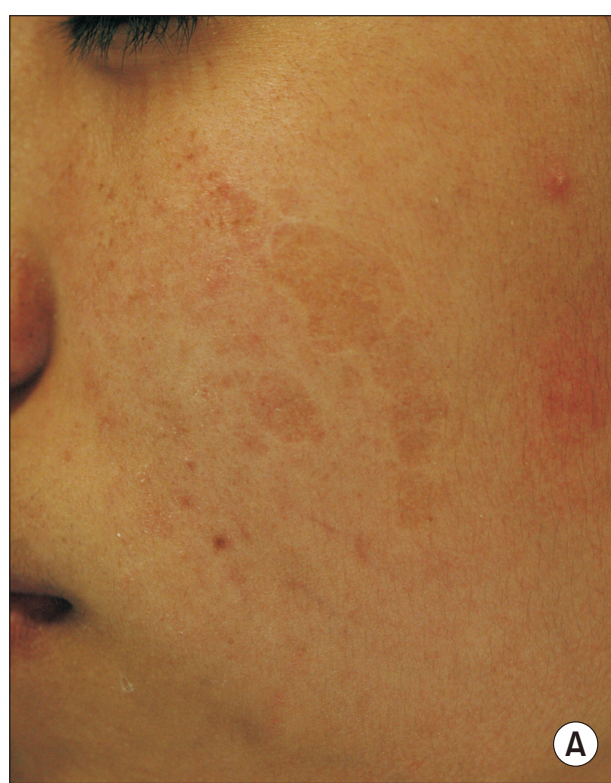

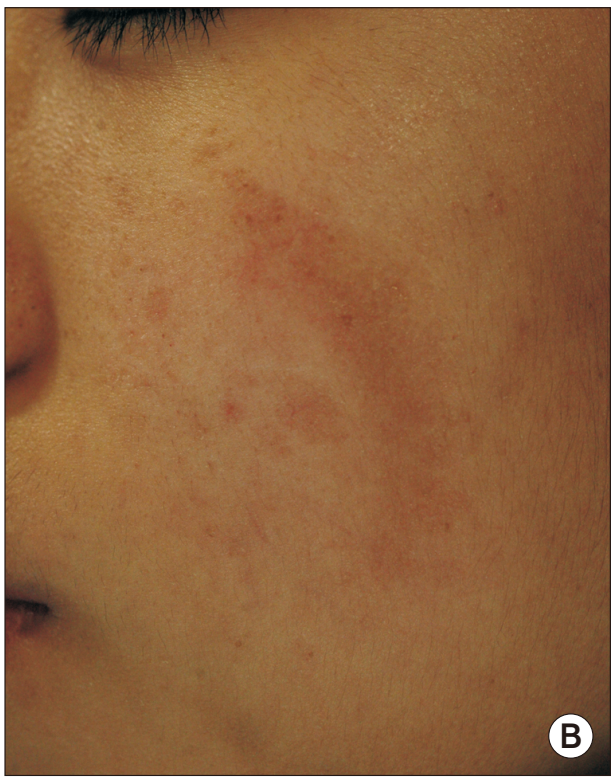

Fig. 1. Case 1. A 24-year-old male patient with herpes simplex virusinduced atrophic scars on the cheek (A) before and (B) two month after two sessions of combined treatment with a non-ablative $1,550-\mathrm{nm}$ fractional erbium (Er):glass fiber laser and an ablative 2,940-nm fractional Er:yttrium aluminum garnet (YAG) laser. 
and surrounding skin at $16 \mathrm{~J} / \mathrm{cm}^{2}$ over four passes with the treatment end-point of pinpoint bleeding. Immediately after the combined treatment, sterile gauze soaked in $0.1 \%$ epinephrine diluted with normal saline was applied on the lesions for hemostasis. Then, antibiotic ointment was applied, and systemic antibiotics or corticosteroids were prophylactically prescribed. Two months after the final treatment, the patient's post-HSV atrophic scars were remarkably improved (Fig. 1B). Although post-treatment erythema was observed, his overall skin tone was notably improved, with which the patient was highly satisfied. Other adverse events were not reported.

\section{Case 2}

A 29-year-old female patient presented with atrophic dyspigmented burn scars along the left lower leg. The lesions had developed from a burn injury from a motorcycle exhaust pipe five years earlier. On her first visitation, the hyper- and hypopigmented atrophic burn scars about the size of a palm were photographed (Fig. 2A). After obtaining written informed consent, three sessions of 1,550-nm fractional Er:glass fiber laser (MOSAIC HP) treatment and two sessions of 2,940-nm fractional Er:YAG laser (ACTION II) treatment were separately delivered to the patient at 4-week intervals. To begin, topical anesthetic cream was applied on the lesions for 1 hour and cleansed with 70\% ethanol. Then, the non-ablative 1,550-nm fractional erbium Er:glass fiber laser was performed at the treatment settings of $60 \mathrm{~mJ}$ on the atrophic scar lesions, a total energy of $0.2 \mathrm{~J}-0.4 \mathrm{~J}$, and a static operating mode using a $4 \mathrm{~cm} \times 4 \mathrm{~cm}$ tip. Immediately after the non-ablative laser treatment, the treated area was cooled with ice packs and antibiotic ointment was applied. Four weeks after the non-ablative treatment, ablative 2,940-nm fractional Er:YAG laser treatment was delivered on the scar lesions and surrounding skin at $16 \mathrm{~J} / \mathrm{cm}^{2}$ over four passes until the treatment end-point of pinpoint bleeding was reached. Immediately after the ablative laser treatment, sterile gauze soaked in $0.1 \%$ epinephrine diluted with normal saline was applied on the lesions for hemostasis. Then, antibiotic ointment was applied, and systemic antibiotics or corticosteroids were prophylactically prescribed. Two months after the final treatment, the atrophic dyspigmented burn scars showed remarkable clinical improvement, and the patient was satisfied with her results (Fig. 2B). Moreover, pronounced clinical improvement in overall skin tone and texture was also noticed without remarkable side effects.

\section{DISCUSSION}

In the two cases reported herein, we found that combined treatment method with a non-ablative 1,550-nm fractional Er:glass fiber laser and an ablative 2,940-nm fractional Er:YAG laser was effective in treating atrophic scars in Asian patients. In the first patient, HSV-induced atrophic scars were treated using the ablative and nonablative lasers on the same day, generating marked clinical improvement without remarkable side effects, except for prolonged erythema. In the second patient, atrophic dyspigmented burn scars were treated with ablative and non-ablative lasers alternately at 4-week intervals. The patient also experienced marked clinical improvement without noticeable adverse events.
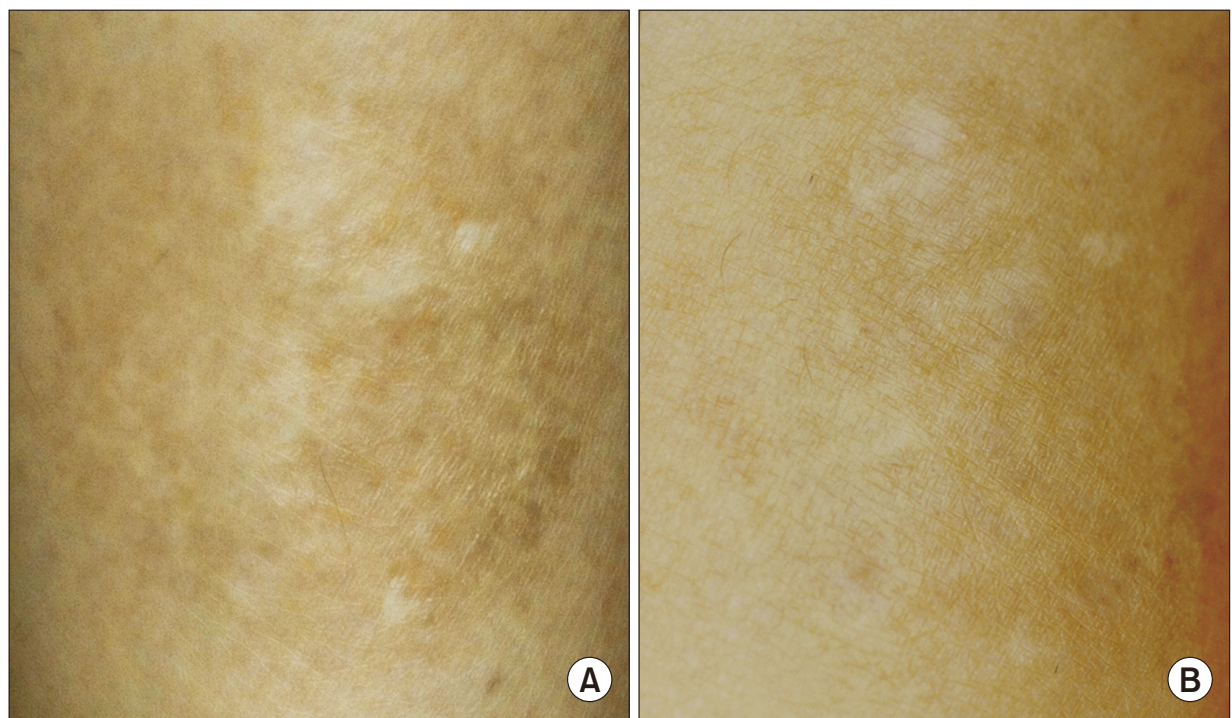

Fig. 2. Case 2. A 29-year-old female patient with an atrophic burn scar on the lower leg (A) before and (B) two months after three sessions of 1,550 $\mathrm{nm}$ fractional Er:glass fiber laser treatment and two sessions of 2,940$\mathrm{nm}$ fractional Er:YAG laser treatment. 
Non-ablative 1,550-nm fractional Er:glass fiber lasers have been shown to generate several necrotic columns on the epidermis and dermis through selective photothermolysis. ${ }^{5}$ In the same study, the 1,550-nm fractional Er:glass fiber lasers effectively induced rearrangement of collagen fibers and stimulation of new collagen production. ${ }^{5}$ Meanwhile, ablative 10,600-nm fractional carbon dioxide lasers have been found to elicit superior effects in tissue shrinkage and increased elasticity, compared to fractional Er:glass fiber lasers. ${ }^{2,4}$ However, considering the pain during the procedure, post-therapy downtime, and the risks of post-inflammatory dyspigmentation and erythema with ablative fractional carbon dioxide lasers, fractional Er:glass fiber lasers are preferred when treating atrophic scars of various origins. ${ }^{4,5}$

Compared to ablative 10,600-nm fractional carbon dioxide lasers, ablative 2,940-nm fractional Er:YAG lasers facilitate induce less thermal injury to the skin due to a higher absorption coefficient to water. ${ }^{6}$ Thereby, 2,940$\mathrm{nm}$ fractional Er:YAG lasers reduce the risk of side effects from ablative laser treatment. Nonetheless, a limited depth of penetration, post-treatment pinpoint bleeding, and the lack of proper thermal stimulation are suggested weak points of 2,940-nm fractional Er:YAG lasers. A previous, randomized study, which compared the efficacy and safety of 2,940-nm fractional Er:YAG lasers and 1,550$\mathrm{nm}$ fractional Er:glass fiber lasers for treating photoaged facial skin, found that 2,940-nm fractional Er:YAG lasers elicited rapid clinical improvement in uneven skin tone and dyspigmentation. ${ }^{6}$ Meanwhile, 1,550-nm fractional Er:glass fiber lasers more effectively improved facial wrinkles with longer lasting dermal remodeling effects, albeit with greater sensations of pain and discomfort during the procedure. ${ }^{6}$

In this study, we combined the use of 1,550-nm fractional Er:glass fiber and 2,940-nm fractional Er:YAG lasers for treating atrophic dyspigmented scars. The 1,550$\mathrm{nm}$ fractional Er:glass fiber laser energy was emitted to generate thermal stimulatory effects in the dermis, without evaporating the epidermis and dermis, and we suggest that the subsequent remodeling of the scar tissue and neocollagenesis generated improvement in the atrophic scars with dermal fibrosis. The 2,940-nm fractional Er:YAG laser treatment was undertaken to stimulate rapid improvement in hyper- and hypopigmented skin tone, as well as impaired skin texture. In our experience, invasive or noninvasive continuous-type radiofrequency devices or invasive pulsed-type radiofrequency devices can also be combined with 1,550-nm fractional Er:glass fiber laser or 2,940-nm fractional Er:YAG laser treatment. Additionally, the combination of low-fluenced Q-switched neodymium:YAG laser treatment, so called laser toning, with or without topical bleaching agents, can be of use in treating accompanying hyperpigmented lesions in atrophic scars.

In conclusion, our cases suggest that combined treatment with a non-ablative 1,550-nm fractional Er:glass fiber laser and an ablative 2,940-nm fractional Er:YAG laser for treating atrophic scars is safe and effective. Although further optimized, prospective studies are needed to confirm our findings, we believe that combined therapy with different fractional lasers provides synergistic therapeutic effects that are of use in treating Asian patients with atrophic scars.

\section{ACKNOWLEDGMENTS}

We would like to thank Anthony Thomas Milliken, ELS (Editing Synthase, Seoul, Korea) for his help with the editing of this manuscript.

\section{REFERENCES}

1. Sage RJ, Lopiccolo MC, Liu A, Mahmoud BH, Tierney EP, Kouba DJ. Subcuticular incision versus naturally sourced porcine collagen filler for acne scars: a randomized split-face comparison. Dermatol Surg 2011;37:426-31.

2. Cho SB, Lee SJ, Cho S, Oh SH, Chung WS, Kang JM, et al. Nonablative 1550-nm erbium-glass and ablative 10 600-nm carbon dioxide fractional lasers for acne scars: a randomized split-face study with blinded response evaluation. J Eur Acad Dermatol Venereol 2010;24:921-5.

3. Zaleski-Larsen LA, Fabi SG, McGraw T, Taylor M. Acne Scar Treatment: A Multimodality Approach Tailored to Scar Type. Dermatol Surg 2016;42:S139-49.

4. Park SH. Fractional laser; NAFL and AFL. Med Lasers 2015:4:1-9.

5. Cho SB, Lee JH, Choi MJ, Lee KY, Oh SH. Efficacy of the fractional photothermolysis system with dynamic operating mode on acne scars and enlarged facial pores. Dermatol Surg 2009;35:108-14.

6. Moon HR, Yun WJ, Lee YJ, Lee MW, Chang S. A prospective, randomized, double-blind comparison of an ablative fractional 2940-nm erbium-doped yttrium aluminum garnet laser with a nonablative fractional 1550-nm erbium-doped glass laser for the treatment of photoaged Asian skin. J Dermatolog Treat 2015;26:551-7. 